\title{
On Profitability and Efficiency of Wireless Mesh Networks
}

Fang Fang, College of Business Administration, Cal State San Marcos, fangfang@csusm.edu; Lili Qiu, Department of Computer Science, The Univ of Texas at Austin, lili@cs.utexas.edu; Andrew Whinston, Dept. IROM, The Univ. of Texas at Austin, abw@uts.cc.utexas.edu.

\section{INTRODUCTION}

The convenience of wireless networking and lightweight handheld devices has led to a large-scale adoption of wireless technologies. Corporations, universities, hospitals, homes, and public places are deploying these networks at a remarkable rate. Even many cities, such as Buffalo (MN), Ripon (CA), Philadelphia (PA), and Portland (OR), have deployed or are planning to deploy city-wide wireless networks. A single-hop wireless network (e.g., current hot-spots) has limited wireless coverage. To enable a large-scale wireless network, wireless mesh networks (WMNs) (e.g., [1, 8, 11]) arise as a promising cost-effective solution that provides high network coverage and low infrastructure cost. In a wireless mesh network, a few Internet transmit access points (ITAPs), serving as gateways to the Internet, are deployed across the neighborhood. Wireless nodes (e.g., houses) are equipped with low-cost antennas, and serve as routers to send traffic for both itself and its neighbors. In this way, a multihop wireless network is formed among wireless nodes to cooperatively route traffic to the Internet through the ITAPs. Such a multihop structure dramatically reduces the number of ITAPs, which is a major cost in deployment.

The promise of wireless mesh networks has attracted lots of research work in the area, ranging from designing MAC protocols (e.g., [7]) to developing routing protocols and routing metrics (e.g., [3, 4]), to studying interactions with TCP (e.g., [5, 6]), to controlling topology via power control (e.g., [9]), channel assignment (e.g., [10]), and directional antennas (e.g., [2]).

In addition to network technologies, another major factor that determines the success of wireless mesh networks is whether there exist viable business models. There is limited research on this problem. In wireless mesh networks, wireless nodes are required to forward traffic for both itself and its neighbors. If the nodes are controlled by self-interested users, they may not efficiently share their capacity to route traffic for other nodes. Such possibility undermines the performance and feasibility of wireless mesh networks. Therefore effective pricing mechanisms need to be developed before the mesh technologies are commercialized.

Motivated by the observations, we develop two pricing mechanisms for non-cooperative wireless mesh networks: a centralized pricing mechanism and a decentralized one. In the centralized pricing mechanism, the service provider needs to monitor and price the traffic originated from every node, while the decentralized scheme leaves the traffic monitoring and pricing to each router. We describe algorithms a service provider uses to efficiently place the ITAPs, and determine the prices. We evaluate the profitability and overall efficiency of the wireless mesh network under the centralized and decentralized pricing mechanisms. As a comparison, we also analyze an alternative structure based on a single-hop wireless network. In such a network, each user can directly communicate with an ITAP, and does not rely on other users for its communication. On the other hand, the singlehop wireless network requires more Internet access points to be deployed, thereby increasing the infrastructure cost. Our analysis has important practical implications to wireless service providers and the future of wireless mesh technologies.

The rest of the paper is organized as follow. In Section 2, we present our models, and develop pricing mechanisms for mesh networks. In Section 3, we describe our evaluation methodology, and present results for realistic mesh networks. Finally we conclude in Section 4.

\section{DEMAND MODEL AND PRICING MECHANISMS}

There is a collection of geographically distributed houses (indexed as $i=1,2, \cdots, N$ ) with potential traffic demands for Internet accesses. Let $\alpha_{i t}$ denote the amount of Internet traffic each 
house $i$ intends to generate (also called intended demands) at time $t$, and $D_{i t}$ be network bandwidth house $i$ obtains (also called the actual demand) at time $t$. Note that $D_{i t} \leq \alpha_{i t}$. $V_{i t}\left(D_{i t}\right)$ and $P_{i t}\left(D_{i t}\right)$ be non-decreasing functions of $D_{i t}$ that represent house $i$ 's value for the bandwidth and the price it is charged at time $t$, respectively. We assume that the utility of a house $i$ follows $u_{i t}\left(D_{i t}, P_{i t}\right)=V_{i t}\left(D_{i t}, \alpha_{i t}\right)-P_{i t}\left(D_{i t}\right)$. We further assume that the value function $V_{i t}$ is increasing and concave with respect to $D_{i t}$. Without loss of generality, we assume that $\frac{\partial^{2} V_{i t}}{\partial D_{i t} \partial \alpha_{i t}}>0$, which indicates that house $i$ values the actual demand increment more at a higher intended demand $\alpha_{i t}$.

Usage-based pricing and fixed pricing are two commonly used charging models. In this paper, we focus on usage-based pricing for the following reasons. First, the usage-based charging is a popular charging model. It also discourages wasting network resources. Second, the fixed pricing gives ISPs less control in regulating users (e.g., in the worst case all the demands are routed through a single user, from whom the ISP can charge). As part of our future work, we plan to directly compare the performance under both charging models. In our analysis, we focus on linear pricing schemes (i.e. $P_{i t}\left(D_{i t}\right)=p_{i t} D_{i t}$ ), because it is one of the simplest usage-based pricing schemes, and users can easily estimate their bills based on their network usage.

Below we introduce both the centralized and decentralized pricing mechanisms, and illustrate the pricing strategy using a simple chain topology with only two houses (see figure 1). House 1 directly connects to ITAP 0 , but house 2 can only connect to house 1 and use house 1 as a relay for Internet access. ITAP 0 is placed by a profit maximizing service provider. For ease of demonstration, we use the value function $V_{i t}=\alpha_{i t} D_{i t}-\frac{1}{2} D_{i t}^{2}$, where $i \in\{1,2\}$. We assume that $\alpha_{i t}$ 's are independently drawn from a distribution function $F(\alpha)$ in the range of $[a, b]$, where $a>\frac{1}{2} b . E(\alpha)$ and $\operatorname{var}(\alpha)$ are the mean and variance of $\alpha_{i t} \mathrm{~s}$, respectively. To evaluate different pricing schemes, we compare the expected profit $(E \Pi)$, and the overall value of the Internet service $E V \triangleq E\left(V_{1}\right)+E\left(V_{2}\right)$.

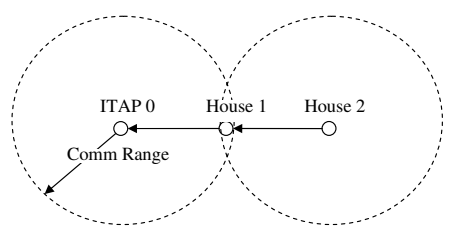

FIGURE 1. A linear topology with 2 houses.

Lemma 1. Given unit price $p_{i t}$, house i's demand $D_{i t}=\alpha_{i t}-p_{i t}$ if $\alpha_{i t} \geq p_{i t}$, and 0 otherwise.

\subsection{Centralized pricing mechanism.}

Dynamically changing price: Suppose both houses subscribe to the service provider, and house 1 agrees to forward traffic for house 2. The profit-maximizing service provider charges a unit price $p_{t}^{c e n}$ for both houses. The price changes according to the realization of $\alpha_{1 t}$ and $\alpha_{2 t}$ to maximize the service provider's expected profit $\Pi_{t}$ at time $t$. That is, $p_{t}^{c e n}=\operatorname{argmax}_{p_{t}} \Pi_{t}=$ $\operatorname{argmax}_{p_{t}}\left\{p_{t}\left[D_{1 t}^{*}\left(p_{t}\right)+D_{2 t}^{*}\left(p_{t}\right)\right]\right\}$.

Proposition 1. With a dynamic centralized pricing mechanism, $p_{t}^{c e n}=\frac{\alpha_{1 t}+\alpha_{2 t}}{4}$. The service provider's expected profit $E \Pi^{c e n}=\frac{(E \alpha)^{2}}{2}+\frac{\operatorname{var}(\alpha)}{4}$ and the expected overall value of the network $E V^{c e n}=\frac{3(E \alpha)^{2}}{4}+\frac{7 \operatorname{var}(\alpha)}{8}$.

This version of the centralized pricing scheme dynamically changes price based on the changing intended traffic demands. To implement such a pricing mechanism, the service provide is required to monitor both the intended demand $\alpha_{i t} \mathrm{~s}$ and the traffic demands $D_{i t} \mathrm{~s}$ from all the users. Such a fine-grained traffic monitoring is expensive and impractical. 
One fixed price: If the ISP cannot obtain the information about the real time demand characteristics $\alpha_{i t}$ s or it has to agree upon a fixed unit price when the customers subscribe, it would choose the one that maximizes its expected profit $E \Pi^{f i x}$. That is, $p^{f i x}=\operatorname{argmax}_{p} p\left\{E\left[D_{1 t}^{*}(p)\right]+E\left[D_{2 t}^{*}(p)\right]\right\}$. Proposition 2. The optimal fixed price $p^{f i x}=\frac{1}{2} E \alpha$, the ISP's expected profit E $\Pi^{f i x}=\frac{1}{2}(E \alpha)^{2}$, and the expected overall value of the service $E V^{f i x}=\frac{3(E \alpha)^{2}}{4}+\operatorname{var}(\alpha)$.

To implement a fixed centralized price, the service provider does not need to know the dynamically changing $\alpha_{i t}$. Only the distribution of $\alpha_{i t} \mathrm{~s}$ is required. In addition, customers prefer a fixed price to an unpredictable price. However, the profit is lower than that of the dynamically changing prices for the same demand level $\alpha_{i t}$ because the service provider cannot adjust the price based on a particular realization of (uncertain) demand. However, the overall value is generally higher when using a fix price scheme. This indicates that the service provider's pricing power may hurt the overall benefit a WMN generates.

2.2. Decentralized pricing mechanism. In such a pricing mechanism, we allow each relay node to determine its own price for routing traffic. This way, the relay node can increase (decrease) the routing price to reduce (increase) the routing traffic when it itself has a large (small) demand to send. However, such a pricing mechanism allows the relays some positive profit and reduce the service provider's potential profitability.

In the example of figure 1 , to decide the optimal price for ITAP0, $p_{0 t}$, the service provider needs to first figure out the pricing strategy house 1 uses to make profit from house 2 . Given the price $p_{0 t}$ and house 2's demand function $\left(\alpha_{2 t}-p_{2 t}\right)^{+}$, House 1's pricing strategy $p_{1 t}^{*}\left(p_{0 t}\right)=$ $\operatorname{argmax}_{p_{1 t}}\left(p_{1 t}-p_{0 t}\right)\left(\alpha_{2 t}-p_{2 t}\right)^{+}$.

Lemma 2. House 1's optimal pricing strategy $p_{1 t}^{*}=\frac{\alpha_{2 t}+p_{0 t}}{2}$ if $\alpha_{2 t} \geq p_{0 t}$ and $p_{1 t}^{*}>\alpha_{2 t}$ otherwise.

Hence, the profit maximizing problem for ITAP0 is:

$$
p_{0 t}^{d e c}=\operatorname{argmax}_{p_{0 t}} \Pi_{t}^{d e c}=\operatorname{argmax}_{p_{0 t}} p_{0 t}\left\{\left(\alpha_{1 t}-p_{0 t}\right)^{+}+\left(\alpha_{2 t}-p_{1 t}^{*}\right)^{+}\right\}
$$

Proposition 3. The ISP's optimal price $p_{0 t}^{\text {dec }}=\frac{1}{3} \alpha_{1 t}+\frac{1}{6} \alpha_{2 t}$, the expected profit $E \Pi=\frac{3}{8}(E \alpha)^{2}+$ $\frac{5}{24} \operatorname{var}(\alpha)$ and the expected overall value $E V=\frac{19}{32}(E \alpha)^{2}+\frac{215}{288} \operatorname{var}(\alpha)$. Meanwhile, the house 1 expect to collect $E \pi_{1}=\frac{9}{16}(E \alpha)^{2}+\frac{61}{144} \operatorname{var}(\alpha)$ profit by serving as a router.

The decentralized pricing scheme requires all nodes in the network (including both the ITAPs and the houses) to constantly monitor the dynamic changing demand of their neighbors. Different from the first version of centralized scheme, in this case an ITAP only needs to monitor the load on its incoming link, and do not need to distinguish and verify how much traffic is originated from its neighbors and how much traffic is relayed by its neighbors for other nodes. Hence, such a coarsegrained traffic monitoring incurs a significantly lower cost, As a tradeoff, part of the profit will be taken away by the routers.

Comparisons of Proposition 1 - 3 shows that the decentralized pricing scheme yields the least value because both ITAP 0 and house 1 can exert pricing power to control the overall network traffic. However, the decentralized pricing scheme has the potential to yield higher profit than the fix price scheme if the variance is large enough.

2.3. Placement of ITAP. If ITAP 0 can be moved from the current location to the place in between the two houses, then both houses can directly communicate with it. This becomes a traditional single hop wireless network. Based on the fact that the service provider aims to maximize profit, we can show that different ITAP placement will not change the profit under the first two pricing schemes. However, both the profit and the customer value increase under the decentralized pricing 
scheme. This is because the placement of ITAP0 changes how traffic is routed in the network, and hence changes the routers' pricing power. This example demonstrates that the placement of ITAPs is especially important to the profit and efficiency of the decentralized pricing scheme. This motivates us to focus on analyzing the decentralized pricing scheme in the following section.

\section{Evaluation Methodology and Results}

In this section, we first describe our evaluation methodology, and then present our evaluation results and explain the intuition behind them.

3.1. Evaluation Methodology. We evaluate the efficiency and profitability of different pricing mechanisms as follow. We randomly place houses in a region of size $R \times R$. There is a wireless link between two houses if and only if their distance is within the wireless communication range $(C R)$. Given a neighborhood layout, finding the optimal placement of ITAPs (i.e., the smallest number of ITAPs that serve the houses) is an NP-hard problem, which means that there is no polynomial algorithm that optimizes ITAP locations unless $\mathrm{P}=$ NP. To achieve efficiency and low computational cost, we determine the location of ITAPs using the following greedy algorithm. In the first iteration, we compute how many houses can be served if we place an ITAP at each of possible locations, and select the one that results in the largest number of served houses. Then in the next iteration, we pick the one that in conjunction with the already chosen ITAP that results in the largest number of served houses. We iterate until all the houses are served. We consider a house is served by an ITAP if it is within $H C$ hops away from the ITAP, where $H C$ is an input parameter specifying the maximum allowed hop count (i.e., the number of links a house traverses before reaching the ITAP). After placing the ITAPs, each house uses the closest ITAP in terms of the number of hop count to access the Internet. The exact route a house uses towards the ITAP is based on the shortest hop count, which is found using Dijkstra's algorithm. The motivation of limiting the hop count between a house and an ITAP and for routing traffic along the path with the smallest hop-count is that wireless network throughput tends to decrease fast with an increasing hop count.

Based on the path, we then decide the real time price $p_{i t}$ each router $i$ uses if the traffic is sent through it. A router chooses a price such that it maximizes the aggregate profit of routing the traffic for all its children. That is, $p_{i t}^{*}=\operatorname{argmax}_{p_{i t}}\left(p_{i t}-p_{\text {parent }}\right) \sum_{j \in\{\text { children of } i\}} D_{j t}\left(p_{i t}\right)$. Similarly each ITAP chooses a price to maximize its aggregate profit except that the price $p_{\text {parent }}=0$.

Proposition 4. Given $p_{\text {parent, }}$, the optimal price for each router $i, p_{i t}=\frac{1}{2}\left(\frac{A_{i t}}{B_{i t}}+p_{\text {parent }}\right)$, where $A_{i}$ and $B_{i}$ are defined as $A_{i} \triangleq \sum_{j \in S_{i}} \frac{\alpha_{j t}}{2^{n_{j}-n_{i}}}$ and $B_{i} \triangleq \sum_{j \in S_{i}} \frac{1}{2^{n_{j}-n_{i}}}$. In the expression, $n_{i}$ and $n_{j}$ denote the length of node $i$ and $j$ 's routing path and $S_{i}$ is the set of node $j$ whose routing path include router $i$ and $\alpha_{j t}<\frac{\sqrt{B_{i t}}-\sqrt{B_{i t}-2^{n_{i}-n_{j}}}}{2^{n_{i}-n_{j}}}\left[\frac{A_{i t}}{\sqrt{B_{i t}}}+\sqrt{B_{i t}-2^{n_{i}-n_{j}}} p_{\text {parent }}\right]$.

To measure the profitability of the decentralized pricing scheme, we mainly focus on the following three measures: (1) the expected revenue for the service provider, which is calculated as the aggregate revenue all the ITAPs collect $\left(E \Pi \triangleq E\left\{\sum_{k \in\{I T A P s\}}\left[p_{k} \cdot D_{k}\right]\right\}\right) ;(2)$ the expected aggregate customer valuation of the WMN $\left(\sum_{i \in\{\text { Houses }\}} E V_{i}\right) ;(3)$ the expected number of ITAPs to be placed, which directly reflects the installation cost of the WMN.

In our evaluation, we vary the following parameters: (1) $N$ : the number of houses; (2) $H C$ : the maximum allowed hop count; (3) $R * R$ : the size of neighborhood. 
3.2. Evaluation Results. We first examine the overall profit as we vary the unit cost of installing an ITAP $(c)$ and the maximum allowed hops $(H C)$. The overall profit is defined as the service provider's expected revenue minus the aggregate cost of installing the ITAPs. As shown in Figure (2), as the unit cost of installing ITAPs increases, the expected overall profit decreases. Its reduction rate is smaller when the number of maximal allowed hop count is larger. This is as what we would expect, since increasing the maximal allowed hop count decreases the number of required ITAPs and hence reduce the installation cost. In addition, the figure also shows that the decrease in the installation cost can overcome the revenue loss of increasing the allowed hops, making the single hop structure suboptimal in some circumstance. This result demonstrates that the mesh technology is cost effectiveness.

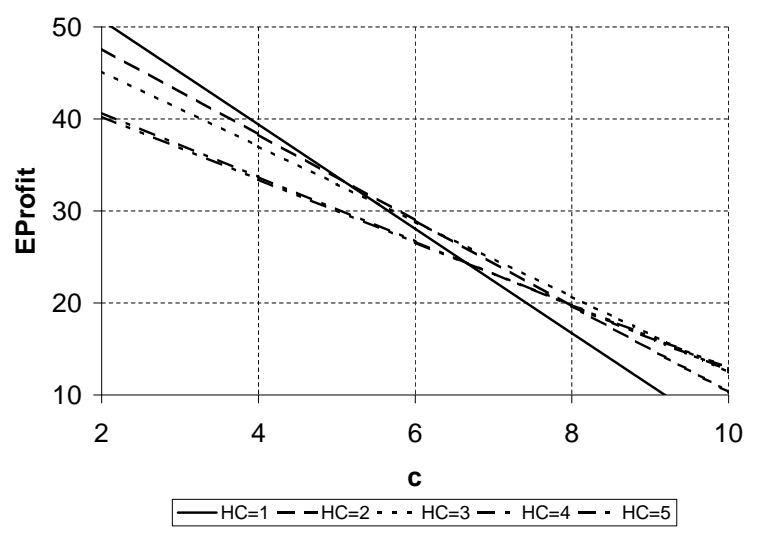

FIGURE 2. Expected profit as the unit cost of installing an ITAP and maximum allowed number of hops change ( $R=70, N=20$, and $\alpha \sim U[3,4])$

Next we study the impact of the region size $R$, which in turn changes network density $\left(N / R^{2}\right)$. In our evaluation, the number of houses is fixed to be 35. Figures 3 (a)-(c) summarize the results. As shown in Figure 3(a), the service providers' revenue first decreases and then increases as $R$ increases when $H C \geq 2$. This trend can be explained as follows. When $R$ is small, every node can easily connect to an ITAP without using many routers. As $R$ initially increases, more nodes need to go through multihop paths in order to reach ITAPs, which makes profit be taken away by the intermediate relay nodes. As $R$ continues to increase, the mesh network becomes disconnected, and at least one ITAP is required in each disconnected component. So more houses are directly connected with an ITAP, which reduces the likelihood of intermediate relay nodes' taking away profit. This is also evident from figure 3(b), which shows that the expected number of ITAPs increases as the density decreases. Meanwhile, the total welfare decreases first and then increase when $H C \geq 2$.

Finally we fix the density of houses (defined as $N / R^{2}$ ) and increase the number of houses. Figures 4(a)-(c) show the change of profitability, the expected number of ITAPs, and customer value, respectively. Since the number of houses varies, we use revenue/house, the number of ITAPs/house, and customer value/house to measure the profitability, infrastructure cost, and efficiency. These measures give us a good idea about the ability of the service provider to make profit from its users and the users' expected efficiency loss due to the decentralized pricing. As the figures show, all the measures decreases as $H C$ increases. This suggests that there is a tradeoff between the profitability (efficiency as well) and the cost saving under decentralized pricing scheme. In all the three figures, we cannot find significant change of profit/house, welfare/house and expected number of ITAPs as the number of houses changes. This indicates that network density is a more important factor than the number of houses that determines profitability and efficiency in a WMN. 


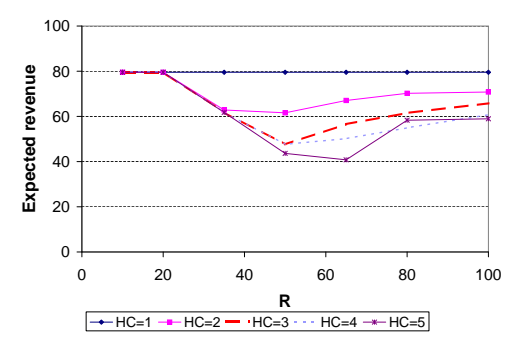

(a)Expected revenue

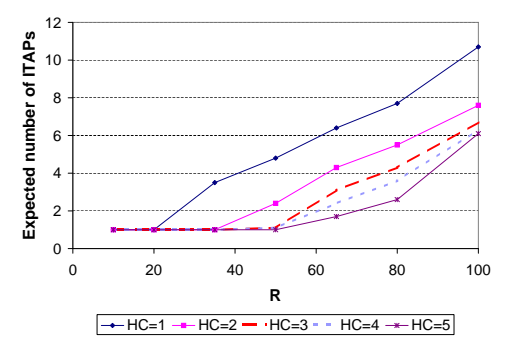

(b)Expected number of ITAPs

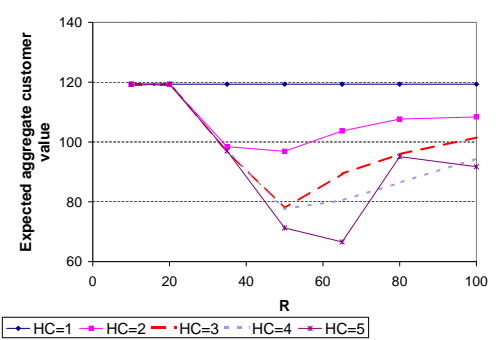

(c) Expected customer value

FIgURE 3. Varying the network density, where $N=35, C R=15$, and $\alpha \sim U[3,4]$.

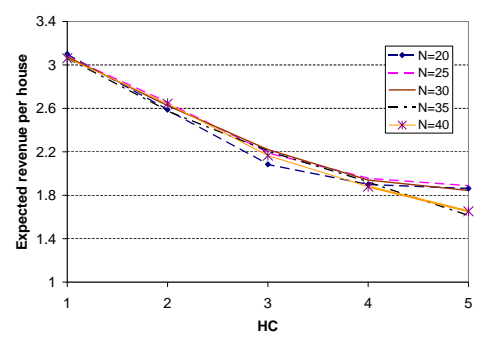

(a)Expected revenue/house
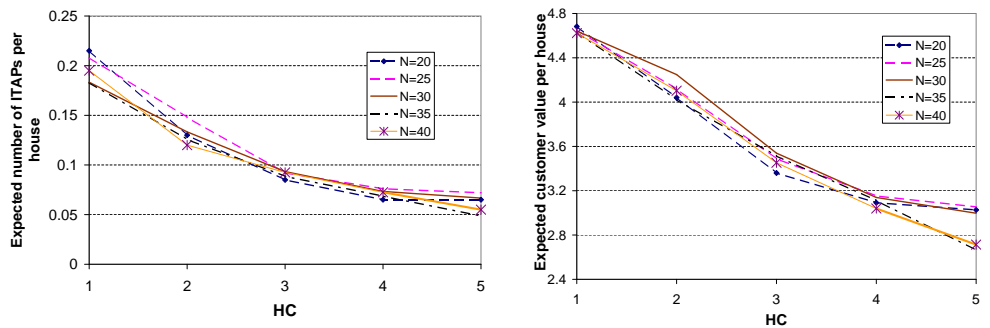

(b)Expected number of ITAPs/house (c) Expected customer value/house FIGURE 4. Varying the number of houses, where $C R=15, N / R^{2}=\frac{1}{125}$, and $\alpha \sim U[3,4]$.

\section{CONCLUSION}

In this paper, we study the potential pricing schemes for implementing emerging wireless mesh networks in a non-cooperative network. We propose several pricing schemes, and analyze their efficiency and profitability. We also evaluate a dynamic decentralized pricing scheme under varying network configurations. Our results show that the efficiency and profitability is sensitive to network density and the maximum number of allowed hop count.

\section{REFERENCES}

[1] 802.11 b community network list. http://www.toaster.net/wireless/community.html.

[2] R. R. Choudhury and N. H. Vaidya. Impact of directional antennas on ad hoc routing. In Proc. of 8th International Conference on Personal Wireless Communication (PWC), September 2003.

[3] D. D. Couto, D. Aguayo, J. Bicket, and R. Morris. A high-throughput path metric for multi-hop wireless routing. In Proc. of ACM MOBICOM '2003, Sept. 2003.

[4] R. Draves, J. Padhye, and B. Zill. Routing in multi-radio, multi-hop wireless mesh networks. In Proc. of ACM MOBICOM, Sept. - Oct. 2004.

[5] M. Gerla, R. Bagrodia, L. Zhang, K. Tang, and L. Wang. TCP over wireless multi-hop protocols: Simulation and experiments. In Proc. of 1999 IEEE International Conference on Communications (ICC), Jun. 1999.

[6] G. Holland and N. Vaidya. Analysis of TCP performance over mobile ad hoc networks. In Proc. of ACM MOBICOM, 1999.

[7] Y. Liu and E. Knightly. Opportunistic fair scheduling over multiple wireless channels. In Proc. of IEEE INFOCOM, 2003.

[8] Self-organizing neighborhood wireless mesh networks. http://www.research.microsoft.com/mesh.

[9] S. Narayanaswamy, V. Kawadia, R. S. Sreenivas, and P. R. Kumar. Power control in ad-hoc networks: Theory, architecture, algorithm and implementation of the compow protocol. In Proc. of European Wireless Conference, 2002.

[10] A. Raniwala, K. Gopalan, and T. Chiueh. Centralized algorithms for multi-channel wireless mesh networks. In Proc. of ACM Mobile Computing and Communications Review (MC2R), April 2004.

[11] MIT Roofnet. http://www.pdos.lcs.mit.edu/roofnet/. 